\title{
Venous thromboembolism and heparin use in COVID-19 patients: juggling between pragmatic choices, suggestions of medical societies and the lack of guidelines
}

\author{
Angelo Porfidia ${ }^{1} \cdot$ Roberto Pola ${ }^{2,3}$ (D) \\ Published online: 4 May 2020 \\ ๑) Springer Science+Business Media, LLC, part of Springer Nature 2020, corrected publication 2020
}

\section{Introduction}

The disease caused by coronavirus SARS-CoV-2, named COVID-19, has become a global emergency. The virus spreads via respiratory droplets and is mainly responsible for infections of the respiratory airways, with potentially fatal pneumonia [1,2]. Although mortality is higher in elderly frail patients presenting with comorbidities, cases of deaths have been reported also in younger age groups [3].

Physicians treating patients with COVID-19 are facing novel and unexpected challenges. One of these is the proper prevention and diagnosis of venous thromboembolism (VTE) and, more generally, how to deal with coagulative activation apparently existing in these patients. In this article, we discuss the many doubts currently existing on the use of heparins and the correct prevention and diagnosis of VTE in COVID-19 patients, with physicians that juggle between pragmatic choices, different suggestions being released on a daily by hospital and medical societies, and the lack of solid evidence or guidelines. Finally, we highlight the need for a call to action by the medical and scientific community.

Roberto Pola

roberto.pola@unicatt.it

1 Division of Internal Medicine, Fondazione Policlinico Universitario A. Gemelli IRCCS Università Cattolica del Sacro Cuore, Rome, Italy

2 Department of Cardiovascular Sciences, Fondazione Policlinico Universitario A. Gemelli IRCCS Università Cattolica del Sacro Cuore, Rome, Italy

3 Istituto di Medicina Interna e Geriatria, 9th Floor, C Wing, Room C907 Policlinico A. Gemelli L.go A. Gemelli 8, Rome 00168, Italy

\section{Coagulative activation and risk of VTE in COVID-19 patients}

Acute infections are associated with a transient increased risk of VTE and subjects hospitalized for COVID-19 pneumonia are the prototypical example of acutely ill medical patients at increased risk of VTE. Indeed, hospitalized COVID-19 patients not only suffer from an acute infection, but also have respiratory failure and are bedridden, or have reduced mobility, due to the need of oxygen supplementation and the isolation imposed by hospital restrictions. The combination of these conditions results in a PADUA Prediction Score $\geq 4$, which corresponds to increased risk for VTE [4]. This risk may be higher, in case of cancer, history of previous VTE, and age $\geq 70$ years [4]. In addition, abnormal coagulation parameters have been reported in hospitalized patients with severe COVID-19 disease [5, 6] and D-dimer levels above $>1000 \mu \mathrm{g} / \mathrm{L}$ have been associated with increased risk of death [7]. It is also known that some patients with severe COVID-19 meet the criteria for disseminated intravascular coagulation (DIC) and display laboratory sings of vascular inflammation [5]. Based on this, one would expect that, in the absence of contraindications, proper thromboprophylaxis is given to all hospitalized patients with COVID-19 pneumonia. A retrospective analysis conducted at Tongji hospital in Wuhan, China, reports that, among 449 patients with severe COVID-19, only 99 $(22 \%)$ received heparin treatment (mainly low molecular weight heparin, LMWH) for 7 days or longer. The 28-day mortality among subjects with Sepsis-Induced-Coagulopathy (sic.) score $\geq 4$, or D-dimer levels $>6$ fold of upper normal limit, was higher in non-heparin than in heparin treated patients [8]. The Authors of this retrospective analysis correctly point out that anticoagulation was seldom used in this population because at the time of its hospitalization there was a poor understanding of the disease, and they state that 
heparin has been increasingly used later during the outbreak of COVID-19 in China.

The use of prophylactic-doses of LMWH is now recommended by the International Society on Thrombosis and Haemostasis (ISTH) for all hospitalized COVID19 patients, unless they have active bleeding or platelet count $<25 \times 10^{9} / \mathrm{L}$ [9]. Likewise, the American Society of Hematology (ASH) states that all hospitalized patients with COVID-19 should receive pharmacologic thromboprophylaxis with LMWH or fondaparinux, unless they are judged to be at increased bleeding risk [10]. However, there is a strong feeling around the medical community that prophylactic doses of anticoagulation might not be sufficient to contrast the hypercoagulable state displayed by many COVID-19 patients in response to a cytokine storm syndrome. In this scenario, some physicians, underlying the anti-inflammatory role of heparin [11, 12], and even its anti-viral potentials [13-15] are considering higher dose of anticoagulation in patients that do not have documented VTE [16, 17]. In any case, it is important to point out that, at the moment, the use of anticoagulation at doses different from those commonly used for thromboprophylaxis is not supported by any published evidence, but only by pragmatic considerations. In particular, the true incidence of VTE in COVID-19 patients that receive pharmacological thromboprophylaxis remains uncertain, as we will discussed below, and data showing improved outcomes in patients treated with therapeutic anticoagulation are lacking. In this puzzling situation, physicians around the world are making decisions on a case by case basis and therapeutic regimens are very heterogeneous from hospital to hospital, even within the same country.

We are also concerned about the fact that nothing is known about the best strategy to use to prevent VTE in the many COVID-19 patients that are treated at home, either before and after hospitalization. One might argue that these subjects are comparable to medical outpatients with minor provoking factors for VTE (i.e. immobility, minor injury, illness, or infection) and that international guidelines suggest not to use VTE prophylaxis in these cases [18]. However, guidelines on this topic have very low level of evidence as they have been indirectly extrapolated from data on acutely ill medical inpatients who received extended prophylaxis upon hospital discharge. In addition, we are doubtful that all patients with COVID-19 who are treated at home are comparable to medical outpatients with minor provoking factors for VTE, especially when they have clinical signs of respiratory infection, fever, cough, and dyspnea. Also, most of these patients are elderly and have reduced mobility, as they are confined in their houses, or even in their bedrooms, in order to avoid spreading the infection to the other family members. They might also have other comorbidities that increase VTE risk, such as cancer, previous VTE, heart failure, and venous insufficiency. Therefore, it is reasonable to hypothesize that at least some of these outpatients would benefit from pharmacological thromboprophylaxis. To support this concept, it is worth mentioning the report published by Danzi and coll., which describes the case of a 75-year-old woman who was hospitalized in Cremona, Italy, after 10 days of fever and a recent onset of dyspnea at home and was diagnosed with severe COVID-19-positive bilateral pneumonia and concomitant acute PE two days after hospital admission [19]. It is also worth mentioning the case of a 28 -year-old man, who, according to the news in the media, died because of PE 10 days after been discharged from a hospital in Trento, Italy, where he had been treated for a confirmed case of COVID19 pneumonia [20]. Of note, both the woman in Cremona and the young man in Trento had no strong predisposing risk factors for VTE, which suggests that the criteria that we classically use to estimate the risk for VTE in outpatients may not apply well to subjects with COVID-19, who might instead have infection per se as precipitant factor for acute VTE, and reduced mobility due to the current restriction rules that have been adopted by many countries to reduce the diffusion of SARS-CoV-2 infection as contributing factor, even when people are not in the hospital. This issue is critical and has been taken into consideration in a recent position paper from the Italian Society on Thrombosis and Haemostasis (SISET), in which it is suggested to maintain thromboprophylaxis at home for 7-14 days after hospital discharge or in the pre-hospital phase, at least in subjects with pre-existing or persisting VTE risk factors [16].

\section{Diagnosis of VTE in COVID-19 patients}

At the moment, very little is known on the exact incidence of VTE among hospitalized patients with COVID-19 pneumonia. This is due to the fact that diagnostic exams cannot be easily performed in these patients, since they must remain in isolation, there is a risk of virus aerosolization, sometimes a lack of proper protective personal equipment, or patients are too unstable. The consequence is that for now there are only two studies published on this issue, both performed on patients assisted in Intensive Care Units (ICU). The first study has been carried out in the Netherlands and reports a remarkable $27 \%$ incidence of VTE (including both DVT and PE), confirmed by either computed tomography pulmonary angiography (CTPA) and/or venous ultrasonography of the legs, on a total of 184 evaluated patients [21]. The second study has been carried out in China and reports a 25\% incidence of DVT, assessed by venous ultrasonography of the legs, on a total of 81 evaluated patients [22]. It is very important to point out that in the Dutch study all patients were receiving at least standard doses of anticoagulant thromboprophylaxis, while in the Chinese study no preventive anticoagulant 
was administered to patients. In addition to these studies, there are two 'preprints' - which are preliminary reports that have not undergone peer review - and case reports. One of these preprints reports 4 cases of deep vein thrombosis (DVT) among 138 COVID-19 patients consecutively admitted to a hospital in China, with a prevalence of $2.9 \%$ [23]. The Authors of this study highlight that 3 of these DVTs were found in critically ill patients, suggesting that the incidence of VTE may be higher in subjects with severe COVID-19. Another preprint reports that, among 1008 patients with COVID-19 pneumonia, 25 underwent CTPA and 10 were found to have PE [24]. Regarding case reports, there is the one published by Danzi and coll, which we have mentioned above [12], and another one published by Xie and coll., which describes the cases of two patients who, after presenting with fever, cough, and dyspnea due to COVID-19 pneumonia, displayed respiratory deterioration associated with increased serum D-dimer level and were diagnosed with acute PE by CTPA [25].

Taken together, these findings support the concept that VTE might complicate the clinical course of COVID-19 pneumonia. Indeed, it is well established that acute PE is a cause of clinical deterioration in viral pneumonias $[26,27]$ and we do not see why COVID-19 pneumonia should make an exception. However, we have evidence from our clinical practice that the diagnostic algorithm that international guidelines recommend for patients with suspected PE, which consists in the execution of CTPA in subjects with high clinical probability of PE independently on D-dimer levels, and in subjects with low or intermediate clinical probability of $\mathrm{PE}$ in case of positive D-dimer test [28], is used very rarely in subjects with COVID-19 pneumonia. This is probably due to the fact that the value of the clinical prediction rules that have been developed to estimate the probability of PE, such as the Geneva score [28], is uncertain in subjects with COVID-19 pneumonia. Nonetheless, we wonder whether such approach might have resulted in missing cases of PE around the world.

Not to mention the fact that many COVID-19 patients stay at home with fever and respiratory symptoms for several days before being hospitalized, as in the case of the 75-year-old woman described by Danzi and coll.,[12] and thus might have VTE already at the moment of hospital admission. It is for these reasons that some hospitals are considering to carry out a systematic screening of deep vein thrombosis (DVT) by means of an ultrasonography of the legs among all hospitalized COVID-19-positive patients. However, a cost-effectiveness analysis of such systematic approach has not been done. We wonder whether ultrasound screening might be more effective if limited to patients with elevated D-dimer levels and/or clinical signs of the disease, as assessed by probability scores (i.e. Wells score). In any case, a screening based only on ultrasound of the legs would not identify 'isolated' PE (i.e. PE without DVT of the legs).

\section{Conclusions}

There are many open questions on the correct diagnosis and management of VTE during the COVID-19 pandemic. Some are the following: What is the prevalence of VTE among patients hospitalized for COVID-19 outside of the ICU? Is there a need for a dedicated scoring system to estimate the risk of VTE among patients with COVID-19? What is the optimal dose of anticoagulation to prevent VTE in COVID19 patients? Should patients treated at home receive pharmacological thromboprophylaxis? Should we screen COVID19 patients for DVT at the moment of hospital admission, or during hospitalization? When should we consider performing CTPA to rule out PE in patients with COVID-19 pneumonia?

We call for a sustained research effort to investigate the issues identified here and reduce the burden of VTE and its consequences in patients with COVID-19. We are aware that randomized clinical trials show the best scientific evidence, but in this moment of emergency and uncertainty, we solicit the medical community to share data and create dedicated registries.

Author contribution Angelo Porfidia and Roberto Pola contributed equally to this article. Both authors discussed, commented, and finally approved the manuscript.

Funding This research received no specific grant from any funding agency in the public, commercial, or not-for-profit sectors.

\section{Compliance with ethical standards}

Conflict of interest The authors declare that they have no conflicts of interest.

\section{References}

1. Huang C, Wang Y, Li X, Ren L, Zhao J, Hu Y, Zhang L, Fan G, Xu J, Gu X, Cheng Z, Yu T, Xia J, Wei Y, Wu W, Xie X, Yin W, Li H, Liu M, Xiao Y, Gao H, Guo L, Xie J, Wang G, Jiang R, Gao Z, Jin Q, Wang J, Cao B (2020) Clinical features of patients infected with 2019 novel coronavirus in Wuhan, China. Lancet 395:497-506

2. World Health Organization. WHO Director-General's opening remarks at the media briefing on COVID-19-11 (March 2020) https://www.who.int/dg/speeches/detail/whodirector-general-sopening-remarks-at-the-media-briefing-on-covid-19---11-march -2020 Accessed 12 March 2020 
3. Liu K, Chen Y, Lin R, Han K (2020) Clinical features of COVID19 in elderly patients A comparison with young and middle-aged patients. J Infect. https://doi.org/10.1016/j.jinf.2020.03.005

4. Barbar S, Noventa F, Rossetto V, Ferrari A, Brandolin B, Perlati M, De Bon E, Tormene D, Pagnan A, Prandoni P (2010) A risk assessment model for the identification of hospitalized medical patients at risk for venous thromboembolism: the Padua Prediction Score. J Thromb Haemost 8:2450-2457

5. Tang N, Li D, Wang X, Sun Z (2020) Abnormal Coagulation parameters are associated with poor prognosis in patients with novel coronavirus pneumonia. J Thromb Haemost 18:844-847

6. Fan BE, Chong VCL, Chan SSW, Lim GH, Lim KGE, Tan GB, Mucheli SS, Kuperan P, Ong KH (2020) Hematologic parameters in patients with COVID-19 infection. Am J Hematol. https://doi. org/10.1002/ajh.25774

7. Zhou F, Yu T, Du R, Fan G, Liu Y, Liu Z, Xiang J, Wang Y, Song B, Gu X, Guan L, Wei Y, Li H, Wu X, Xu J, Tu S, Zhang Y, Chen H, Cao B (2020) Clinical course and risk factors for mortality of adult inpatients with COVID-19 in Wuhan, China: a retrospective cohort study. Lancet 395:1054-1062

8. Tang N, Bai H, Chen X, Gong J, Li D, Sun Z (2020) Anticoagulant treatment is associated with decreased mortality in severe coronavirus disease 2019 patients with coagulopathy. J Thromb Haemost. https://doi.org/10.1111/jth.14817

9. Thachil J, Tang N, Gando S, Falanga A, Cattaneo M, Levi M, Clark C, Iba T (2020) ISTH interim guidance on recognition and management of coagulopathy in COVID-19. J Thromb Hemost. https://doi.org/10.1111/jth.14810

10. Baumann Kreuziger L, Lee A, Garcia D, Cuker A, Cushman M, Connors JM (2020) COVID-19 and VTE/Anticoagulation: Frequently Asked Questions. www.hematology.org/covid-19/covid -19-and-vte-anticoagulation

11. Poterucha TJ, Libby P, Goldhaber SZ (2017) More than an anticoagulant: Do heparins have direct anti-inflammatory effects? Thromb Haemost 117:437-444

12. Young E (2008) The anti-inflammatory effects of heparin and related compounds. Thromb Res 122:743-752

13. Shukla D, Spear PG (2001) Herpesviruses and heparan sulfate: an intimate relationship in aid of viral entry. J Clin Invest 108:503-510

14. Ghezzi S, Cooper L, Rubio A, Pagani I, Capobianchi MR, Ippolito G, Pelletier J, Meneghetti MCZ, Lima MA, Skidmore MA, Broccoli V, Yates EA, Vicenzi E (2017) Heparin prevents Zika virus induced-cytopathic effects in human neural progenitor cells. Antiviral Res 140:13-17

15. Vicenzi E, Canducci F, Pinna D, Mancini N, Carletti S, Lazzarin A, Bordignon C, Poli G, Clementi M (2004) Coronaviridae and SARS-associated coronavirus strain HSR1. Emerg Infect Dis 10:413-418

16. Marietta M, Ageno W, Artoni A, De Candia E, Gresele P, Marchetti M, Marcucci R, Tripodi A (2020) COVID-19 and haemostasis: a position paper from Italian Society on Thrombosis and Haemostasis (SISET). Blood Transfus. https://doi. org/10.2450/2020.0083-20

17. Thachil $\mathrm{J}$ (2020) The versatile heparin in COVID-19. J Thromb Haemost https://doi.org/10.1111/jth.14821
18. Schünemann HJ, Cushman M, Burnett AE, Kahn SR, BeyerWestendorf J, Spencer F, Rezende S, Zakai NA, Bauer K, Dentali F, Lansing J, Balduzzi S, Darzi A, Morgano GP, Neumann I, Nieuwlaat R, Yepes-Nuñez JJ, Zhang Y, Wiercioch W (2018) American Society of Hematology 2018 guidelines for management of venous thromboembolism: prophylaxis for hospitalized and nonhospitalized medical patients. Blood Adv 2:3198-3225

19. Danzi GB, Loffi M, Galeazzi G, Gherbesi E (2020) Acute pulmonary embolism and COVID-19 pneumonia: a random association? Eur Heart J. https://doi.org/10.1093/eurheartj/ehaa254

20. http://www.ladige.it/news/cronaca/2020/03/31/altri-17-decessicovid-ultime-24-ore

21. Kloka FA, Kruipb MJ, van der Meerc NJ, Arbousd MS, Gommerse DA, Kantf KM, Kapteina FH, van Paassend J, Stalsa MA, Huismana MV, Endemane H (2020) Incidence of thrombotic complications in critically ill ICU patients with COVID-19. Thromb Res. https://doi.org/10.1016/j.thromres.2020.04.013

22. Cui S, Chen S, Li X, Liu S, Wang F (2020) Prevalence of venous thromboembolism in patients with severe novel coronavirus pneumonia. J Thromb Haemost. https://doi.org/10.1111/jth.14830

23. Xu J, Wang L, Zhao L, Li F, Liu J, Zhang L, Li Q, Gu J, Liang S, Zhao Q, Liu J (2020) Risk assessment of venous thromboembolism and bleeding in COVID-19 patients. Respiratory Research. https://doi.org/10.21203/rs.3.rs-18340/v1

24. Chen J, Wang X, Zhang S, Liu B, Xiaoqing W, Wang Y, Wang X, Yang M, Jianqing S, Yuanliang X (2020) Findings of Acute Pulmonary Embolism in COVID-19 Patients. Available at SSRN. https://doi.org/10.2139/ssrn.3548771

25. Xie Y, Wang X, Yang P (2020) Zhang S (2020) COVID-19 Complicated by Acute Pulmonary Embolism. Radiology Cardiothoracic Imaging 2(2):e200067. https://doi.org/10.1148/ryct.20202 00067

26. Jolobe OMP (2019) Similarities Between Community-Acquired Pneumonia and Pulmonary Embolism. Am J Med 132:e863. https ://doi.org/10.1016/j.amjmed.2019.03.002

27. Ishiguro T, Matsuo K, Fujii S, Takayanagi N (2019) Acute thrombotic vascular events complicating influenza-associated pneumonia. Respir Med Case Rep 28:100884. https://doi.org/10.1016/j. rmcr.2019.100884

28. Konstantinides SV, Meyer G, Becattini C, Bueno H, Geersing GJ, Harjola VP, Huisman MV, Humbert M, Jennings CS, Jiménez D, Kucher N, Lang IM, Lankeit M, Lorusso R, Mazzolai L, Meneveau N, Ní Áinle F, Prandoni P, Pruszczyk P, Righini M, Torbicki A, Van Belle E, Zamorano JL, ESC Scientific Document Group (2020) 2019 ESC Guidelines for the diagnosis and management of acute pulmonary embolism developed in collaboration with the European Respiratory Society (ERS). Eur Heart J 41:543-603

Publisher's Note Springer Nature remains neutral with regard to jurisdictional claims in published maps and institutional affiliations. 